\title{
Visualizing International Author Collaboration Characteristics and Topic Burst on Transportation Management: A Bibliometric Analysis
} \author{
Mei-Yu Tu${ }^{1}$, Tsair-Wei Chien ${ }^{2,3}$ Willy Chou $^{4,5}$ \\ ${ }^{1}$ Department of Nutrition, Chi Mei Medical Center, Tainan, Taiwan \\ ${ }^{2}$ Department of Hospital and Health Care Administration, Chia Nan University of Pharmacy and Science, Tainan, \\ Taiwan \\ ${ }^{3}$ Research Department, Chi Mei Medical Center, Tainan, Taiwan \\ ${ }^{4}$ Department of physical medicine and rehabilitation, Chi Mei medical center, Tainan, Taiwan \\ ${ }^{5}$ Department of Recreation and Health-Care Management \& Institute of recreation Industry Management, Chia Nan \\ University of Pharmacy, Tainan, Taiwan
}

Running title: Author collaboration and keyword on transportation management

\begin{abstract}
Background: Whether the international author collaboration and keywords on the topic of transportation management has been changed in the last several decades remains unclear. Along with the big data and API(allocation programming interface) emerged as a field of research with increasing attention being paid to it by scientific researchers and a rapid increase in related literature being reported using the bibliometric analysis, the international author collaboration and keywords should be explored to analyze the current state of research, including publication outputs, in-depth collaboration characteristics and keyword topics of transportation management research.

Methods: The authors collected two published papers in Medline library and downloaded their 206 similar articles without duplication since 1977. Various statistical techniques and bibliometric measures were employed, including publication growth analysis; journal distribution; and collaboration network analysis at the author country/area collaboration level. The visualization maps of international author collaboration and burst terms were drawn on Google maps using social network analysis(SNA) and cluster analysis. Gini coefficient(GC) was applied to measure inequality of density indices among clusters.
\end{abstract}

Results: A total of 208 bibliographic records on transportation management were collected. The earliest paper was published in 1977, with the number of papers sharply rising at the inflection point of the year 2014. We found that (1) the most number of papers on the topic of transportation management are from the U.S.( 43,27.04\%), Spain(21,13.21\%), and China(18,11.32\%); (2) the most linked keywords are organization \& administration, analysis, education, and statistics \& numerical data, and trends; (3) keyword networks presents lower GC that author collaborations among their respective clusters.

Conclusions: The collaboration of international authors on transportation management is not tight and stable. The focus of research topics on transportation management is centralized(Gini $=0.48$ ) more than that of author collaboration (Gini=0.33). Our study might provide a potential guide for future research on the topic of transportation management.

Keywords: Collaboration Characteristics; Topic Bursts; Transportation Management; Bibliometric Analysis; Gini Coefficient

\section{Background}

A transportation management system (TMS) is a subset of supply chain management concerning transportation operations and may be part of an enterprise(or others like a hospital) resource planning system. A TMS usually refers to 
a process related to warehouse/distribution module. A typical scenario would include both inbound (procurement) and outbound (shipping) orders to be evaluated by the TMS Planning Module.

With continued economic and societal development worldwide, the traditional TMS has increasingly failed to satisfy enterprise(or others like a hospital) in providing efficient delivery services. It should be noted that numerous constraints and barriers exist to providing high-quality, accessible, and timely health services, especially in low-resource settings.

Given the importance of TMS, some scientific researchers have focused on reviewing the related literature to identify the characteristics and status of TMS in recent years. However, much of this effort in author collaboration used by bibliometric analysis hazs only considered specific subfields drawn from descriptive analysis and systematic reviews ${ }^{[1-3]}$ using social network analysis(SNA).

However, It is hard to find the relationship between multiple entities in which we are interested instead of those counts or frequencies of interesting items. That is, the sense of their correlation can yield much more information. For instance, in Big data when many customers purchase their goods by placing them in a shopping cart (which is similar to the author collaboration or the keywords in a published paper), we are interested in the co-occurrence for any two or more goods sold in a store. An apocryphal story was often told to tell us the concept of co-occurrence that is about beer and diaper sales which usually goes along with a strong correlation on Friday ${ }^{[4-6]}$. Many data scientists have developed ways to discover new knowledge from the vast quantities of increasingly available information ${ }^{[7]}$, especially applying social network analysis (SNA $)^{[3-5]}$ to big data analysis.

Other reviews have concentrated on the analysis of $\mathrm{MTS}^{[6]}$ and the method ${ }^{[9]}$. Few focus on the topic of international author collaboration. As of June 12, 2018, only two papers ${ }^{[10,11]}$ were found by the keyword of "Transportation Management" in the title in Medline library. There are four topics that intrigue us to study, including (1) which countries/areas contribute most in the paper publication of TMS? (2) which keywords are more frequently found in TMS? (3) whether the phenomena of author collaboration and keywords are equally distributed among entities or clusters?

Admittedly, authorship collaboration using SNA is an example illustrated by many authors in recent years ${ }^{[3]}$ because co-authors among researchers form a type of social network. Whether the keyword network in TMS earns an equally important impact is interesting to explore. We are thus to apply SNA to explore the features in TMS from published papers ${ }^{[10,11]}$ and their related papers we observed in Medline library.

Google maps have provided users to gain an overall geospatial visualization ${ }^{[12,13]}$. However, few were found in Medline library when searching the keyword google map [Title] on June 12, 2018. Many papers ${ }^{[3-5,14]}$ have conducted studies on co-author collaboration. However, none display these results combined with Google maps and social network analysis.

Our aims are to apply Gini coefficient $(\mathrm{GC})^{[15]}$ to the pattern of international author collaboration in TMS on the following topics: (i) nation distribution over the years; (ii) keywords distribution and their frequencies in TMS; (3) the phenomena of author collaboration and keywords equally distributed among entities or clusters..

\section{Methods}

\subsection{Data sources}

We programed Microsoft Excel visual basic for applications (VBA) modules to extract abstracts and their corresponding data related to coauthors and medical subject heading(MESH) terms for each article on June 12, 2018, by the keywords of "Transportation Management" in the title in Medline library since 1977. Only those related to the articles searched above using the functionality of similar articles provided by Pubmed and labeled with Journal Article were included. Others like those marked with Published Erratum, Editorial or without author nation name were excluded from this study. A total of 208 eligible abstracts were obtained from Medline in which 159 with nation name for the $1^{\text {st }}$ author were found.

\subsection{Data arrangement to fit SNA requirement}


Before visualizing our results using SNA, we organized data in compliance with the format and guidelines defined by Pajek software ${ }^{[16]}$. Microsoft Excel VBA routines were used to deal with data fitting to the SNA requirement.

\subsection{Graphical representations to report}

(1) Author nations and their relations

Two tables (including columns for publication years and rows for the $1^{\text {st }}$ author nations and density coefficient for author networks) were made to represent the distribution of nations and journals in addiction and clinical research. The bigger bubble means, the number of the nodes (i.e., nations, authors or keywords in this study). The wider line indicates, the stronger relations between the two ties. Community clusters with a closer relation are filled with different colors in the bubbles.

(2) Keywords to present the research domain

If our keywords represent the mainstream topics on the topic of TMS, the stronger relations between the two keywords can be highlighted and linked by SNA. Like the concept of co-occurrence about beer and diaper sales. The presentation for the bubble and line is interpreted similarly to the previous section. The density coefficients for the keywords using MESH terms among clusters were analyzed in this study.

\subsection{Statistical tools and data analyses}

Google Maps and SNA Pajek software were used to visualize international author collaborations and keywords with the keywords of TMS. Author-made Excel VBA modules were applied to organize data. Cluster coefficient ${ }^{[13]}$ represents the density of a network as below:

$$
\frac{\text { number of closed troplets }}{\text { number of possible connected triplets of nodes }}=\frac{\sum_{i=1}^{n} \text { other_two_nodes } i_{-} \text {connected }}{\sum_{i=1}^{n}\left(m_{i} *\left(m_{i}-1\right) / 2\right.}
$$

, whereas $n=$ the number of nodes in a network and $m=$ the number of other connected nodes with a specific ego node. A significant lever $(>1.96)$ is defined by $\mathrm{t}$-value as the formula $\left[=\mathrm{cc} * \sqrt{ }\left[(\mathrm{n}-2) /\left(1-\mathrm{cc}^{2}\right)\right]\right.$.

In contrast, $\mathrm{E}-\mathrm{I}$ index is defined by the formula, where $\mathrm{EL}=$ the number of external friendship links and $\mathrm{IL}=\mathrm{the}$ number of internal friendship links ${ }^{[17,18]}$. The negative E-I index means a coherence cluster in existence. Similarly, the higher CC indicates many members are other linked members' friends. Density is defined as the ratio of the linked members over all possible linked members.

\section{Results}

\subsection{Growth of Literature}

By the above search strategy and the cleaned data obtained, we found that the earliest paper on transportation management collected by Medline library was published in 1977. The publication output of TMS, from 1977 to 2018, is presented in Figure 1, indicating that the number of papers concerning TMS in Medline has risen yearly and produced from 1 in 1977 to 26 in 2018. Since the beginning of 2014, it should be recorded that the number of TMS-related publications has increased considerably.

The cumulative annual number of publications has continually grown from 2 to 48 (shown in Figure 1). A literature exponential growth curve was obtained by a direct fit to the equation (R2>0.81). The time of the inflection point of the growth curve is at 2014 .

\subsection{Author nations and their relations}

A total of 159 eligible papers with complete author nations based on journal article since 1977 are shown in Table 1. We can see that the most number of papers are from the U.S.( 43,27.04\%), Spain(21,13.21\%), and China(18, $11.32 \%)$. The trend in the number of publications is present in the column of growth in Table 1. The top three continents are Asia, Europe, and South America.

The diagram shown by SNA and Google Maps in Figure 1 displays the author's collaboration among nations based on transportation management. Overall, the highest productive nations are from the US, Spain, and Chien, see Figure 1. 
Any nation collaborated with other nations are shown with a blue line. Interested authors are recommended to click the bubble of interest to see details on a website at reference ${ }^{[19]}$.

\begin{tabular}{|c|c|c|c|c|c|c|c|c|c|c|c|c|c|c|}
\hline Continent & 1977-08 & 2009 & 2010 & 2011 & 2012 & 2013 & 2014 & 2015 & 2016 & 2017 & 2018 & Total & $\%$ & Growth \\
\hline AFRICA & 1 & & & & & 1 & & & 1 & & & 3 & 1.89 & 0.31 \\
\hline Egypt & 0 & & & & & 1 & & & & & & 1 & 0.63 & 0 \\
\hline Ghana & 1 & & & & & & & & & & & 1 & 0.63 & \\
\hline Morocco & 0 & & & & & & & & 1 & & & 1 & 0.63 & 0.41 \\
\hline Asia & 4 & & 1 & 1 & 3 & 3 & 2 & 1 & 6 & 5 & 4 & 30 & 18.87 & 0.77 \\
\hline China & 1 & & & & 1 & 1 & 2 & 1 & 5 & 4 & 3 & 18 & 11.32 & 0.86 \\
\hline Taiwan & 0 & & & & 1 & 2 & & & & & & 3 & 1.89 & -0.06 \\
\hline India & 1 & & & 1 & & & & & & & & 2 & 1.26 & -0.27 \\
\hline Turkey & 0 & & 1 & & & & & & & & 1 & 2 & 1.26 & -0.41 \\
\hline Hong Kong & 0 & & & & 1 & & & & & & & 1 & 0.63 & -0.14 \\
\hline Israel & 1 & & & & & & & & & & & 1 & 0.63 & \\
\hline Pakistan & 0 & & & & & & & & 1 & & & 1 & 0.63 & 0.41 \\
\hline Philippines & 1 & & & & & & & & & & & 1 & 0.63 & \\
\hline South Korea & 0 & & & & & & & & & 1 & & 1 & 0.63 & 0.55 \\
\hline EUROPE & 6 & 3 & 1 & & 2 & 2 & 5 & 10 & 13 & 11 & 12 & 65 & 40.88 & 0.86 \\
\hline Spain & 1 & & & & 2 & & 1 & 3 & 5 & 7 & 2 & 21 & 13.21 & 0.86 \\
\hline U.K & 2 & 1 & & & & & 2 & 6 & 1 & 1 & 1 & 14 & 8.81 & 0.4 \\
\hline Italy & 0 & & 1 & & & & & & 2 & 2 & 3 & 8 & 5.03 & 0.57 \\
\hline Netherlands & 0 & 2 & & & & & & & 2 & & & 4 & 2.52 & -0.1 \\
\hline Austria & 1 & & & & & & & & & & 1 & 2 & 1.26 & \\
\hline Germany & 0 & & & & & 1 & 1 & & & & & 2 & 1.26 & 0.1 \\
\hline Greece & 1 & & & & & & & 1 & & & & 2 & 1.26 & 0.27 \\
\hline Romania & 0 & & & & & & & & & & 2 & 2 & 1.26 & \\
\hline Subtotal & 1 & 0 & 0 & 0 & 0 & 1 & 1 & 0 & 3 & 1 & 3 & 10 & $\begin{array}{r}6.29 \\
-\end{array}$ & 0.64 \\
\hline N. AMERICA & 25 & 2 & 3 & 4 & 1 & 2 & 4 & 2 & 2 & 4 & 3 & 52 & 32.7 & 0.16 \\
\hline U.S & 23 & 2 & 3 & 3 & 1 & 2 & 3 & 1 & 2 & 2 & 1 & 43 & 27.04 & -0.29 \\
\hline Canada & 2 & & & 1 & & & 1 & 1 & & 2 & & 7 & 4.4 & 0.57 \\
\hline Mexico & 0 & & & & & & & & & & 2 & 2 & 1.26 & \\
\hline OCEANIA & 2 & & & & & & 1 & 1 & 2 & & & 6 & 3.77 & 0.57 \\
\hline Australia & 2 & & & & & & 1 & 1 & 2 & & & 6 & 3.77 & 0.57 \\
\hline S. AMERICA & 0 & & & & & & & 2 & & 1 & & 3 & 1.89 & 0.52 \\
\hline Brazil & 0 & & & & & & & 1 & & 1 & & 2 & 1.26 & 0.62 \\
\hline Colombia & 0 & & & & & & & 1 & & & & 1 & 0.63 & 0.27 \\
\hline Total & 38 & 5 & 5 & 5 & 6 & 8 & 12 & 16 & 24 & 21 & 19 & 159 & 100 & 0.92 \\
\hline
\end{tabular}

Table 1. Paper outputs across continents/country/area over the years 

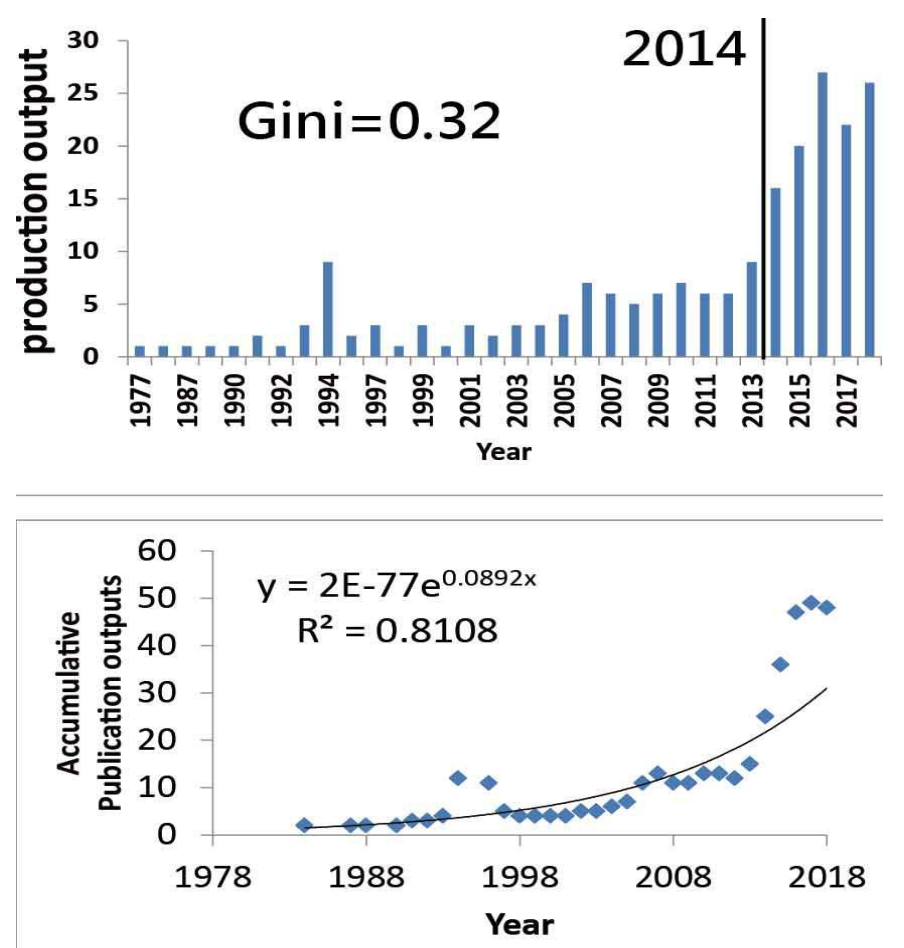

Figure 1; Publication outputs over the years for papers collected in Medline library on the topic of transportation management.

\subsection{Keywords to present the research domain of transportation management}

The most linked keywords are organization \& administration, analysis, education, and statistics \& numerical data, and trends, see Figure 3 or click it on the reference ${ }^{[20]}$. We can see that the keywords consist of many clusters with different cluster coefficients.

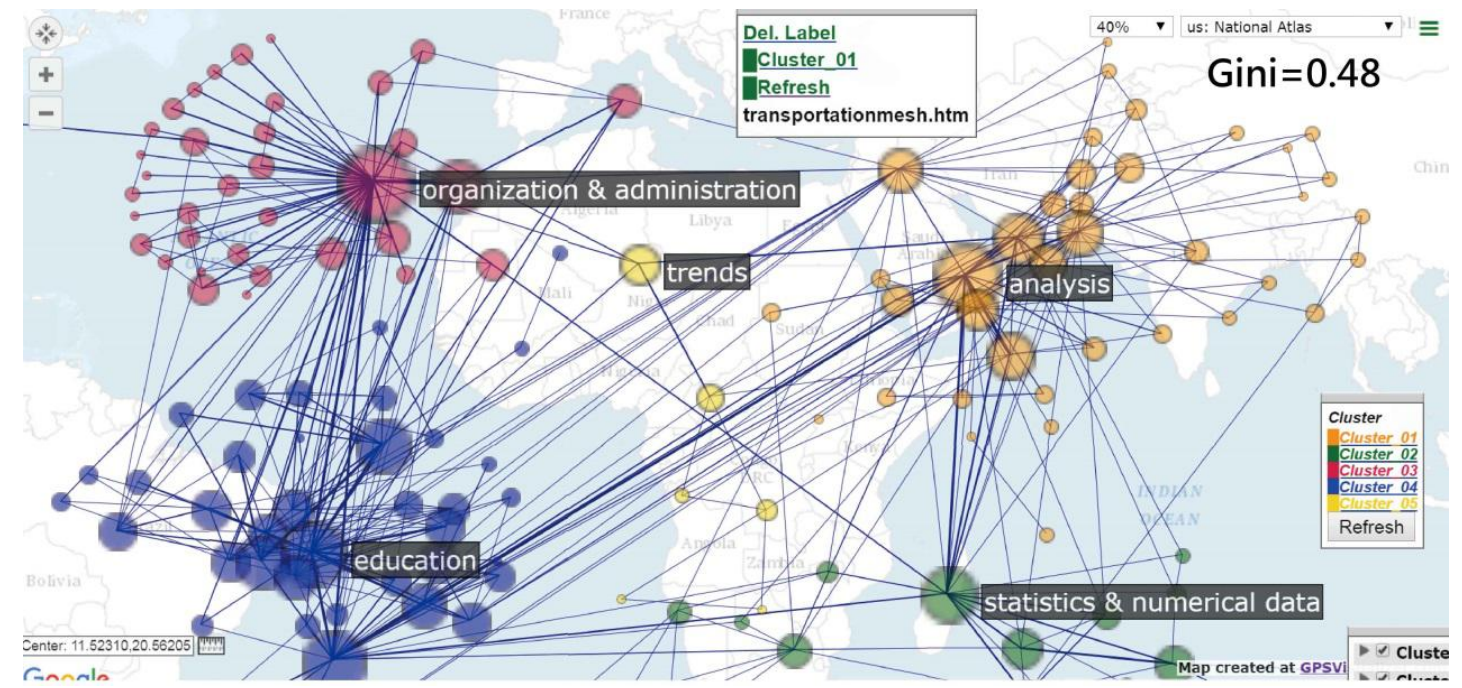

Figure 3; MESh terms regarding papers collected in Medline library on the topic of transportation management.

\subsection{Cluster density coefficients in networks}

Each cluster has its cluster coefficient to represent the density of a network. We found that author clusters earn an approximately lower CC that those in keyword clusters (Table 2 and Table 3). Only the cluster coefficient for the keyword of education has a significant effect in comparison with a substantial $t$-value $(>2.0)$, indicating all but the education networks have the feature of low density in a network. Keyword networks present lower GC that author collaborations among their respective clusters (Table 2 and Table 3).. 


\begin{tabular}{|c|c|c|c|c|c|c|c|c|}
\hline $\mathrm{CC}$ & $\mathbf{t}$ & UNWD & WD & EI & $\mathbf{n}$ & Degree & Weighted & Name \\
\hline & & 1 & 1 & 0 & 2 & 1 & 1 & U.S \\
\hline & & & & 1 & 1 & 0 & 0 & Spain \\
\hline & & & & 1 & 1 & 0 & 0 & China \\
\hline 0.43 & 0.82 & 0.5 & 0.5 & -0.33 & 5 & 5 & 5 & U.K \\
\hline 1 & & 1 & 1 & -0.71 & 3 & 3 & 3 & Italy \\
\hline 0.60 & 1.06 & 0.67 & 0.67 & -0.45 & 4 & 4 & 4 & Australia \\
\hline \multirow[t]{2}{*}{0} & 0 & 0.67 & 0.67 & -1 & 3 & 2 & 2 & France \\
\hline & & 1 & 1 & -1 & 2 & 1 & 1 & Pakistan \\
\hline 0.40 & 0.44 & 0.25 & 0.25 & 0.14 & 0.18 & 0.30 & 0.30 & Gini \\
\hline
\end{tabular}

Table 2. Density coefficients for author collaboration based on country or area

Note. $\mathrm{CC}=$ cluster coefficient; UNWD = unweighted density $=$ the unique number of linkages between nations / possible nations(i.e., $\mathrm{n}(\mathrm{n}-1) / 2)$; $\mathrm{WD}=$ weighed density $=$ the multi-repetition number of linkages/possible numbers; Gini $=$ Gini coefficient; EI=E-I index $=($ Internal linkages - External linkages $) /($ Internal linkages + External linkages $)$.

\begin{tabular}{lllllllll}
$\mathbf{C C}$ & $\mathbf{t}$ & UNWD & WD & EI & $\mathbf{n}$ & Degree & Weighted & Name \\
\hline 0.11 & 0.65 & 0.1 & 0.27 & -0.53 & 36 & 61 & 167 & organization \& administration \\
0.28 & 1.75 & 0.13 & 0.22 & -0.54 & 38 & 88 & 158 & analysis \\
0.33 & $2.21 *$ & 0.14 & 0.31 & -0.59 & 42 & 118 & 268 & education \\
0.43 & 1.91 & 0.22 & 0.36 & -0.42 & 18 & 33 & 55 & statistics \& numerical data \\
0.50 & 1.15 & 0.47 & 0.67 & -0.27 & 6 & 7 & 10 & trends \\
\hline 0.2 & 0.15 & 0.35 & 0.21 & 0.09 & 0.17 & 0.23 & 0.24 & Gini coefficient \\
\hline
\end{tabular}

\section{Discussion}

Table 3. Density coefficients for MESH terms based on country or area $* \mathrm{p}<0.05$

This study found that (1) the most number of papers on the topic of transportation management are from the U.S.( 43,27.04\%), Spain(21,13.21\%), and China(18, 11.32\%); (2) the most linked keywords are organization \& administration, analysis, education, and statistics \& numerical data, and trends; (3) keyword networks presents lower GC that author collaborations among their respective clusters.

\subsection{What This Adds to What Was Known}

Many previous types of research ${ }^{[1-4]}$ have inspected coauthor collaboration using social network analysis. The results (the most number of articles in transportation management are from the U.S., Spain, and Chien) are similar to the findings that dominant nations in science come from the U.S. and Europe ${ }^{[21,22]}$. We see a sharp increase in trend for China presenting in this study which is similar to the previous study ${ }^{[14]}$. We showed a novel method incorporating SNA with Google maps to explore the data of publication in TMS. It can be seen that visual representations provide readers with a quick glance at the research results which are rare in the literature. Traditionally, it is very hard to observe the association of two or more symptoms or ties together appeared in a network at the moment.

All entities can be compared with each other on the dashboard of Google Maps. We can see that many links are connecting two nations(Figure 2). Such a network can be defined as a collaboration pattern which results are similar to the previous study ${ }^{[5]}$. Accordingly, the researchers have a high level of international author collaboration on the topic of transportation management, which is consistent with the previous studies on investigating scientific collaboration of Iranian Psychology and Psychiatry Researchers ${ }^{[23,24]}$. 


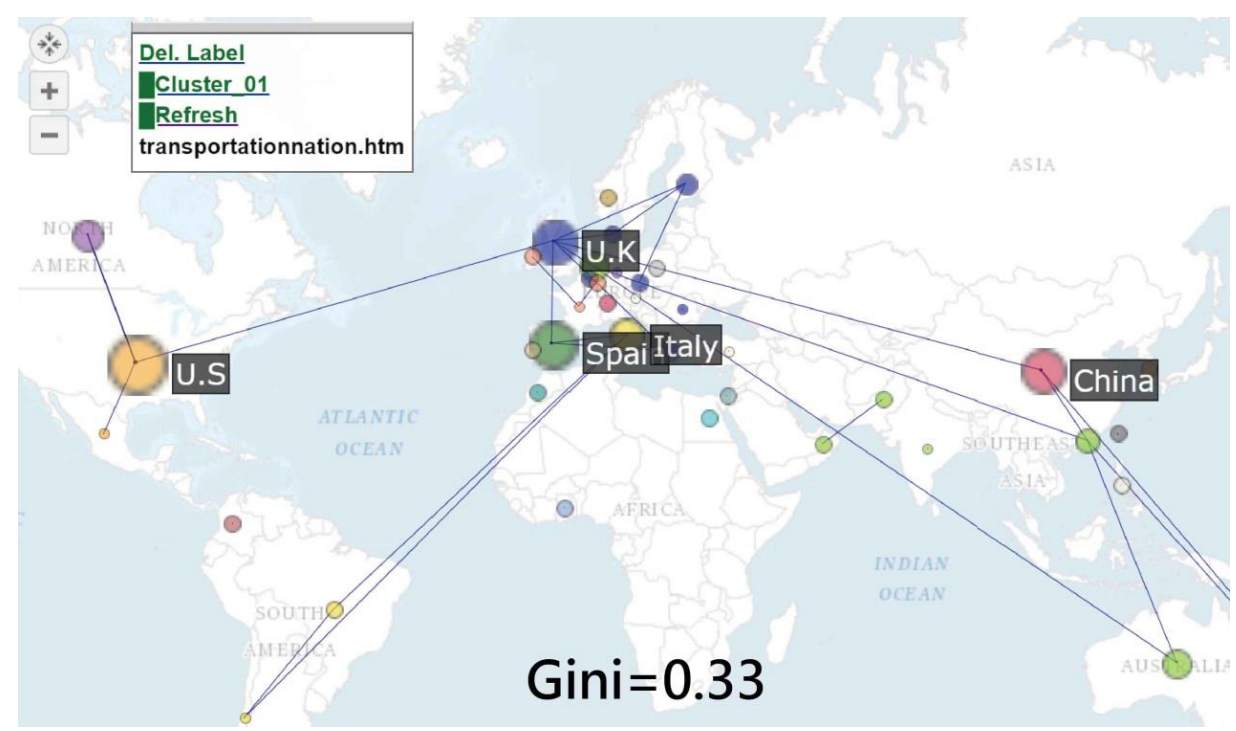

Figure 2; Author country/area distribution for papers collected in Medline library on the topic of transportation management.

There are 1,084 papers with the keyword of "social network analysis" in the paper title when searching Medline on December 21, 2017, in which two papers ${ }^{[25,26]}$ incorporated MeSH into SNA to disclose relevant knowledge to readers. However, no such papers have incorporated Google maps link as we did in the current study. The CCs we illustrated in Table 2 and three are called overall CCs. The highest value represents the closer networks, as shown in figure 2 . It can be seen that three countries of Italy, Brazil, and Chile construct a close relation $(\mathrm{CC}=1)$. In contrast, France has no any three countries connecting in its cluster. Accordingly, CC equals zero, EI equals $-1.0(=(0-2) /(0+2)$, indicating two countries(i.e., Luxembourg and Ireland) constructing a cluster, but none connecting to outside clusters.

\subsection{What It Implies and What Should Be Changed?}

Scientific publication is one of the objective measurements to evaluate the achievements of a medical specialty or discipline ${ }^{[27]}$. It is worth combining SNA and Google Maps to disclose knowledge and information to the readers for reference in the future.

Many algorithms and measures (or indicators) have been developed using SNA to graphically explore data ${ }^{[1-3]}$. This kind of data can identify the interested target entry(e.g., TMS in this study) with paper of selected topics; It means that the core subject can be analyzed using the centrality measure ${ }^{[1-3,28]}$ yielded by SNA.

\subsection{Strengths of This Study}

The way we incorporated SNA with Google Maps is unique in comparison with others ${ }^{[1-3]}$. The network density can be replaced by $\mathrm{CC}$ used in this study. Another strength and feature for this study are that Google Maps are sophisticatedly used and linked in references ${ }^{[19-20]}$ for each interested topic, the reader can manipulate the link by their ways as a dashboard to understand the feature of the author collaboration or the keyword relationship. The nation distribution in Figure 2 is an easily understood feature of international author collaborations on TMS. One picture is worth ten thousand words. We hope following studies can report other types of information using Google API(application programming interface) to readers in the future.

\subsection{Limitations and Future study}

The interpretation and generalization of the conclusions should be cautious. First, the data were extracted from Medline library. It is worth noting that any generalization should be made in the similar fields of paper contents, particularly we merely including papers collected in Medline.

Second, although the data were extracted from Medline and were carefully dealt with in every linkage as correctly as possible, the originally downloaded contexts including some errors in symbols which might affect the resulting reports in this study may be present.

Third, there are many algorithms used for SNA. We merely applied community cluster and density with weighted 
degrees in Figures. Any changes made along with algorithm will present different pattern and inference making.

Fourth, the social network analysis is not subject to the Pajeck software we used in this study, Others such as Ucinet $^{[29]}$ and Gephi ${ }^{[30]}$ are suggested to readers for use in the future study.

\section{Conclusion}

Social network analysis provides wide and deep insight into the relationships among nations and coauthors. The results can provide readers with a concept of diagram for readers to understand the feature on the topic of transportation management.

\section{Conflicts of Interest}

The authors have declared no conflicts of interest.

\section{References}

1. Sadoughi F, Valinejadi A, Shirazi MS, Khademi R.Social Network Analysis of Iranian Researchers on Medical Parasitology: A 41 Year Co-Authorship Survey.Iran J Parasitol. 2016;11(2):204-212.

2. Osareh F, Khademi R, Rostami MK, Shirazi MS. Co-authorship Network Structure Analysis of Iranian Researchers' scientific outputs from 1991 to 2013 based on the Social Science Citation Index (SSCI). Collnet J Scientometr Info Manag. 2014; 8 (2): 263- 71.

3. Liu X, Bollen J, Nelson ML, Van de Sompel H. Co-authorship networks in the digital library research community. Info Process Manag. 2005; 41 (6): 1462- 80.

4. Domingos p. A few useful things to know about machine learning. Communications of the ACM 2012; 55:10,78-87.

5. Verhoef PC, Kooge E, Walk N. Creating Value with Big Data Analytics: Making Smarter Marketing Decisions. London: Routledge, 2016.

6. Power DJ..What is the "true story" about data mining, beer and diapers? DSS News. 2017/3/20 available at https://goo.gl/1FtGft

7. Morris MA, Saboury B, Burkett B, Gao J, Siegel EL. Reinventing Radiology: Big Data and the Future of Medical Imaging. J Thorac Imaging. 2018 Jan;33(1):4-16.

8. Asari S, Kaneshiro T, Hosoda T, inventors; Panasonic Corp, assignee. Transportation management system. United States patent US 2005; 6,975,248.

9. Jauffred F, Ahmed K, Arminio S, Desai H, Johar P, McGregor R, Pluta M, Wilson C, inventors; MANHATTAN ASSOCIATES, assignee. Transportation management system and method for shipment planning optimization. United States patent application US 2005; 11/083,337.

10. Masek P, Masek J, Frantik P, Fujdiak R, Ometov A, Hosek J, Andreev S, Mlynek P, Misurec J.A Harmonized Perspective on Transportation Management in Smart Cities: The Novel IoT-Driven Environment for Road Traffic Modeling. Sensors (Basel). 2016 Nov 8;16(11). pii: E1872.

11. Schneider S.Transportation management of neonatal - pediatric transports.Kinderkrankenschwester. 2015 Jun;34(6):217-20 (in German).

12. Dasgupta S, Vaughan AS, Kramer MR, Sanchez TH, Sullivan PS. Use of a Google Map Tool Embedded in an Internet Survey Instrument: Is it a Valid and Reliable Alternative to Geocoded Address Data?JMIR Res Protoc. 2014 Apr 10;3(2):e24.

13. Kobayashi S, Fujioka T, Tanaka Y, Inoue M, Niho Y, Miyoshi A. A geographical information system using the Google Map API for guidance to referral hospitals. J Med Syst. 2010 Dec;34(6):1157-60.

14. Shen L, Xiong B, Li W, Lan F, Evans R, Zhang W. Visualizing Collaboration Characteristics and Topic Burst on International Mobile Health Research: Bibliometric Analysis.JMIR Mhealth Uhealth. 2018 Jun 5;6(6):e135.

15. Gini C. Concentration and dependency ratios (in Italian). English translation in Rivista di Politica Economica 1997; $87,769-789$.

16. de Nooy W, Mrvar A, Batagelj V. Exploratory Social Network Analysis With Pajek: Revised and Expanded, 2nd edn. New York, NY: Cambridge University Press, 2011.

17. Phan TG, Beare R, Chen J, Clissold B, Ly J, Singhal S, Ma H, Srikanth V. Googling Service Boundaries for Endovascular Clot Retrieval Hub Hospitals in a Metropolitan Setting: Proof-of-Concept Study.Stroke. 2017;48(5):1353-1361

18. Krackhardt D, Stern RN. Informal networks and organizational crises:An experimental simulation. Social Psychology Quarterly 1988; 51,123-140.

19. Chien TW. Google Maps on the topic of collaboration in transportation management. 2018/6/12 available at http://www.healthup.org.tw/gps/transportationnation.htm

20. Chien TW. Google Maps on MESH terms in transportation management. 2018/6/12 available at http://www.healthup.org.tw/gps/transportationmesh.htm 
21. Leydesdorff L, Wagner C, Park HW, Adams J.International collaboration in science: the global map and the network.CoRR abs/1301.0801 (2013)

22. Glänzel W, Schlemmer B. National research profiles in a changing Europe (1983-2003) An exploratory study of sectoral characteristics in the Triple Helix. Scientometrics 2007; 70(2): 267-275.

23. Erfanmanesh MA, Rohani VA, Basirian Jahromi R, Gholamhosseinzadeh Z. Investigating Scientific Collaboration of Iranian Psychology and Psychiatry Researchers. J Info Process Manag.2013; 29 (1): 137- 63.

24. Osareh F, Norouzi Chakoli A, Keshvari M. Co-authorship of Iranian researchers in science, social science, art and humanities citation indexes in the web of science between 2000 and 2006. J Info Sci Technol.2010; 25 (4): 573 95.

25. Hu SK, Huang J, Hong WD, Du XJ, Jin R, Lin TS. The 50 Most-cited Articles in Gastroenterology and Hepatology from Mainland China.Pak J Med Sci.2017; 33(1): 215-220.

26. Coelho DH, Edelmayer LW, Fenton JE. A century of citation classics in otolaryngology-head and neck surgery journals revisited. Laryngoscope.2014;.124(6): 1358-1362.

27. Chang HT, Lin MH, Hwang IH, Chen TJ, Lin HC, Hou MC, Hwang SJ. Scientific publications in gastroenterology and hepatology in Taiwan: An analysis of Web of Science from 1993 to 2013.J Chin Med Assoc.2017; 80(2): 80-85.

28. Chien TW, Chang Y, Wang HY.Understanding the productive author who published papers in medicine using National Health Insurance Database: A systematic review and meta-analysis.Medicine (Baltimore). 2018 Feb;97(8):e9967.

29. Borgatti SP, Everett MG, Freeman LC. Ucinet for Windows: Software for Social Network Analysis. Harvard, MA: Analytic Technologies 2002.

30. Bastian M, Heymann S, Jacomy M. Gephi: an open source software for exploring and manipulating networks. International AAAI Conference on Weblogs and Social Media,2009. 